\title{
Linfoma no Hodgkin primario extranodal de musculatura masticatoria. A propósito de un caso
}

\section{Primary extranodal non-Hodgkin lymphoma of the masticatory muscles. A case report}

\author{
Constanza Bulboa F. ${ }^{1}$, Ruth Orellana F. ${ }^{2}$, Jordi Branera P. ${ }^{3}$, Eduardo Neumann . $^{1}$
}

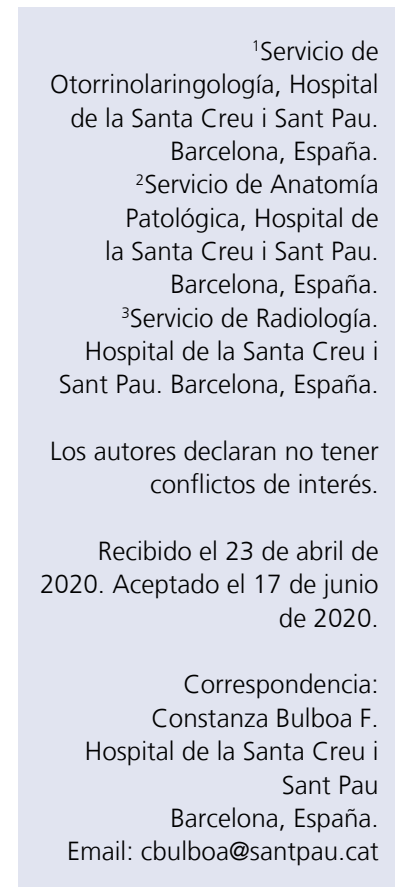

\begin{abstract}
Resumen
Entre el 25\% a 40\% de los linfomas no Hodgkin (LNH) surgen en ubicaciones extranodales. La afectación de los tejidos blandos por el LNH es infrecuente y el linfoma de origen primario del músculo esquelético es aún más inusual. Los músculos mayormente afectados son los de las extremidades, pelvis, región glútea y con menor frecuencia los músculos de cabeza y cuello. En este artículo se presenta nuestra experiencia sobre un caso de linfoma no Hodgkin primario extranodal del músculo masetero, que fue tratado con quimioterapia combinada tipo R-CHOP con buena respuesta. Se revisan en la literatura las características clínicas de esta patología, los criterios diagnósticos y el tratamiento en este tipo de linfoma.
\end{abstract}

Palabras claves: linfoma no Hodgkin, extranodal, espacio masticatorio.

\begin{abstract}
Around 20\% to $45 \%$ of non-Hodgkin lymphomas (NHL) develop in extranodal locations. Soft tissue involvement is rare and skeletal muscle affection is a far more unusual NHL presentation. In this singular scenario, upper and lower extremity muscles are mostly affected, especially those from the pelvic and gluteal area and less frequent of the musculature from the head and neck region. Therefore, we here in report a case of an extranodal NHL of the masseter muscle. Treatment regime was based on R-CHOP combined chemotherapy resulting in a favorable outcome. Diagnostic criteria and clinical characteristics, and treatment of this type of lymphoma are reviewed in this article.
\end{abstract}

Keywords: non-Hodking lymphoma, extranodal, masticatory space.

\section{Introducción}

El linfoma es una neoplasia maligna del sistema linfático, que se caracteriza por una proliferación de las células linfoides o de sus precursores $^{1,2}$. Corresponde al 3\% del total de los tumores malignos, posicionándose como la tercera neoplasia maligna más común. Los linfomas presentan diferentes comportamientos y grados de agresividad, en función del tipo histológico. Se pueden dividir en dos grandes grupos: linfoma de Hodgkin (LH) y linfoma no Hodgkin $(\mathrm{LNH})$, siendo más frecuente este último, con un $80 \%$ a $90 \%$ del total de los linfomas ${ }^{2}$.
Nos centraremos en los linfomas a nivel de cabeza y cuello, los cuales corresponden al 2,2\% del total de las neoplasias malignas de esta zona, siendo sólo superada por los tumores epiteliales ${ }^{1}$. Como dijimos, el LNH es el más frecuente, y a nivel maxilofacial no es la excepción, siendo más común la estirpe de células B3, como es nuestro caso clínico. El origen del LH es generalmente en los ganglios linfáticos, principalmente los que se ubican a nivel cervical y mediastínico; y sólo entre un $1 \%$ a $4 \%$ se presentan en áreas extranodales. Esto es muy diferente a lo que ocurre en el LNH que se presenta a nivel extranodal entre $25 \%$ a $40 \%$ de los casos $^{2,3}$. Dentro de las zonas 
extranodales, y a nivel de cabeza/cuello, se afectan los senos paranasales, cavidad oral, mandíbula, glándula salival, y menos comunes son el espacio parafaríngeo o masticatorio ${ }^{4}$.

La afectación extranodal del LNH en el músculo esquelético es poco frecuente, no obstante, los músculos más afectados son los músculos glúteos y pélvicos, como resultado de diseminación hematógena o linfática, o por extensión por contigüidad de los ganglios linfáticos o huesos involucrados. En la literatura se describe la afectación a nivel muscular secundaria, ocurre sólo en el 1,4\% de los casos, con un $0,3 \%$ en la enfermedad de Hodgkin y $1,1 \%$ en LNH. El linfoma muscular primario es aún menos común, Travis y cols. informaron ocho casos de linfoma primario muscular de 7.000 linfomas en un período de 10 años $(0,11 \%)^{5}$.

En los países occidentales, se ha observado en los últimos años un aumento en la tasa de incidencia anual de LNH a expensas de territorios extranodales. Esto se debe probablemente a la inmunodeficiencia, enfermedades autoinmunes, infecciones, exposición a pesticidas/ solventes químicos y a la susceptibilidad genética ${ }^{6}$. A continuación, se describe el caso de un paciente con un linfoma no Hodgkin primario extranodal del músculo masetero. Se revisan en la literatura las características clínicas de esta patología, los criterios diagnósticos y el tratamiento en este tipo de linfoma.

\section{Caso Clínico}

Presentamos el caso de una mujer de 63 años, sin antecedentes patológicos de interés, que acudió por aumento de volumen en mejilla izquierda, de 4 meses de evolución, que se asociaba a otalgia refleja y dolor durante la masticación. En la exploración física se observó una masa de partes blandas de aproximadamente 5 $\mathrm{cm}$, que deformaba la hemicara izquierda, dura y fija a mandíbula. No se observó compromiso de pares craneales, no se palpó adenopatías cervicales ni se asoció síntomas sistémicos. Se realizó resonancia magnética craneal que informó una lesión en el espacio masticatorio que afectaba al músculo masetero y pterigoideo medial; y que se extiende a la rama mandibular izquierda sin destrucción de la cortical ósea (Figura 1). Se realizó biopsia guiada por tomografía computada (TC) con aguja gruesa de lesión maseterina izquierda, por vía externa, sin incidentes.

El estudio histológico de la muestra informó de una proliferación neoplásica constituida por linfocitos de tamaño intermedio-grande, con estudio inmunohistoquímico positivo para CD20, CD79a y CD30, y focal para BCL6. Negatividad para CD3, CD5, BCL-2, CD10, MUM-1, CD23, ciclina-D1, ALK y queratina AE1/AE3 (Figura 2).

No se detecta hibridación in situ del mRNA del virus Epstein-Barr. Los hallazgos morfológicos y el perfil inmunohistoquímico son concordantes con el diagnóstico de linfoma $\mathrm{B}$ de alto grado, CD30 positivo. El caso clínico es valorado por equipo de hematología donde se realizó estudio hematológico y bioquímico, donde sólo destacó un leve aumento de la proteína beta-2-microglobulina. El estudio de PET-TC descartó afectación en otra parte del cuerpo. Se indica iniciar quimioterapia combinada de rituximab, ciclofosfamida, hidroxidaunorubicina, sulfato de vincristina (oncovin) y prednisona. (R-CHOP), con buena respuesta, logrando su remisión completa. En seguimiento hace más de un año libre de

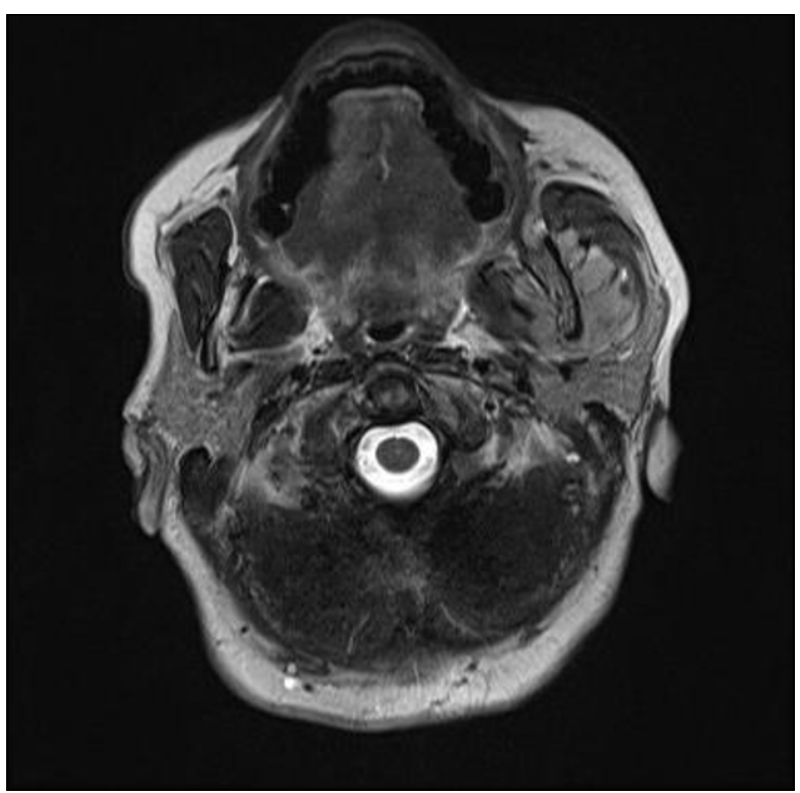

Figura 1. Resonancia magnética (RM) craneal: lesión en el espacio masticatorio que afecta al músculo masetero y pterigoideo medio; y que se extiende rama mandibular izquierda sin destrucción de cortical ósea. 


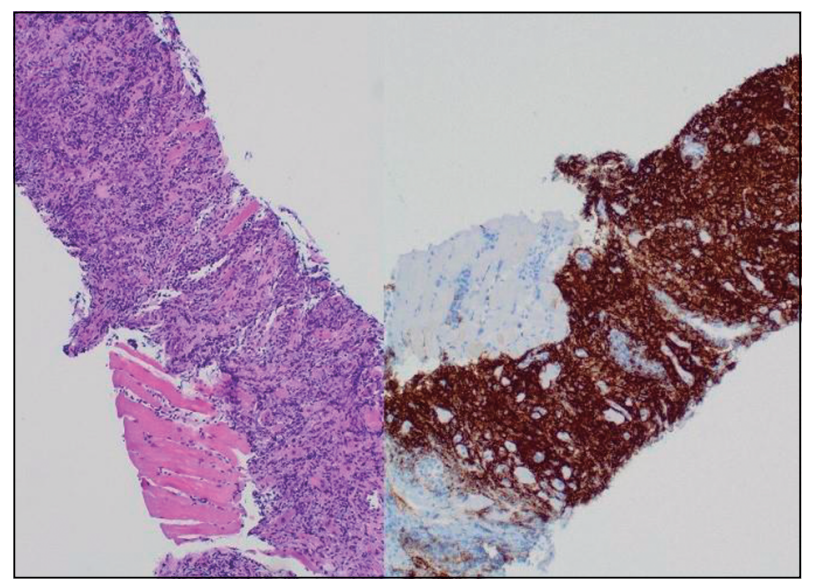

Figura 2. A la izquierda se observa infiltración de músculo esquelético por una proliferación neoplásica de linfocitos de tamaño grande. A la derecha se muestra tinción positiva difusa para CD20.

enfermedad y con una funcionalidad de musculatura masticatoria y ósea correcta.

\section{Discusión}

El LNH puede presentarse en territorios extranodales entre un $25 \%$ a $40 \%$ de los casos totales de linfomas. La ubicación a nivel de cabeza y cuello es la segunda zona del cuerpo más frecuente a nivel LNH extranodal con $11 \%-33 \%^{1}$. Los sitios más comunes extranodales de aparición son: anillo de Waldeyer, suelo de la boca, glándulas salivales, mucosa bucal, senos paranasales y hueso ${ }^{7}$.

En una revisión sistemática de la literatura realizada por Silva y cols. se describió las principales manifestaciones orales del linfoma, destacando el dolor, la inflamación y la sensación de parestesia a nivel facial. En cuanto al género, se encontró un pequeño porcentaje más de hombres afectados que mujeres. El rango de edad del diagnóstico de LNH fue amplio, encontrándose desde los 2-96 años, sin embargo, fue más frecuente en mayores de 50 años ${ }^{1}$. Con respecto a la ubicación a nivel muscular, un estudio realizado por de Samuel y cols. realizó una revisión de más 6.000 pacientes con linfoma durante 15 años. Los autores seleccionaron los casos con infiltración muscular $y$, posteriormente, se excluyeron los que presentaban compromiso muscular por extensión de una adenopatía adyacente o de los otros tejidos blandos. Sólo ocho pacientes se identificaron como LNH primario del músculo, siendo principalmente de pelvis y extremidades inferiores ${ }^{8}$.

El espacio masticador fue descrito por primera vez en detalle por Coller e Yglesias, $y$ la patología que afectaba principalmente a este territorio eran las infecciones. Adyacente y anterior al espacio masticador, se encuentra el espacio bucal; el espacio parotídeo en la parte posterior y el espacio parafaríngeo hacia medial. Los tumores que se originan en el espacio masticador son raros, mientras que las metástasis o la afectación por tumores adyacentes son más comunes ${ }^{10}$. En una revisión realizada por Liapi y cols. se encontraron sólo 6 casos de LNH primario muscular a nivel de la cabeza/ cuello, cinco de estos casos involucraron los músculos de la masticación ${ }^{8}$.

Con respecto a los factores de riesgo se ha observado que los pacientes VIH positivos tienen $60 \%$ más posibilidades de desarrollar un LNH que la población general y la afectación primaria de linfoma a nivel muscular puede verse en $8,8 \%{ }^{8}$. En nuestro caso la paciente no presentaba factores de riesgo. Dentro de los criterios diagnósticos que consideraron Lee y cols. para definir los casos como LNH primario de músculo fueron: la confirmación histológica de este subtipo, la ausencia de compromiso sistémico (estudio con radiografía de tórax, TC de tórax, abdomen y pelvis) y dentro de los hallazgos radiológicos se debía observar un compromiso muscular previo a las anormalidades óseas o que los excedía en gran medida. Estos criterios se cumplen en nuestro paciente ${ }^{11}$.

Por otro lado, los hallazgos radiológicos en la resonancia magnética del LNH que se asocian a la afectación del músculo esquelético se han descrito como hiperintenso o isointenso en relación con el tejido muscular normal en la secuencia $\mathrm{T} 1$ e hiperintensa en la secuencia $\mathrm{T} 2^{4,11}$. Las pruebas de imagen son un pilar importante en el diagnóstico, sin embargo, es fundamental realizar una biopsia que nos permitirá confirmar el diagnóstico y clasificar el tipo de LNH, con el fin de aplicar el tratamiento más adecuado según el subtipo ${ }^{12}$. En nuestro caso, la enfermedad estaba limitada a los músculos del espacio masticatorio. Tampo- 
co se encontraba afecto el espacio parotídeo. No hubo lesiones cutáneas asociadas ni extensión a la cavidad oral o tracto nasosinusal, tampoco compromiso de los pares craneales. No presentaba síntomas B típicos como fiebre, pérdida de peso ni sudoración nocturna.

Según algunos reportes de casos clínicos analizados, se ha observado que el tratamiento se basa en quimioterapia combinada, radioterapia más quimioterapia o con sólo radioterapia con relativa buena respuesta ${ }^{1,8,4}$. Sin embargo, no existe un tratamiento específico para el LNH primario extranodal muscular, aunque se trata con terapias similares a los linfomas de origen nodal, que dependen de la histología, el estadio clínico y las características del paciente ${ }^{1,8}$.

\section{Conclusión}

El linfoma no Hodgkin primario extranodal de los músculos de cabeza/cuello, es una entidad inusual ${ }^{12}$. En pacientes que presenten aumento de volumen a nivel muscular unilateral o bilateral de cabeza/cuello, se debe sospechar linfoma dentro de los diagnósticos diferenciales. Las técnicas especiales que se realizan a la biopsia, como son los marcadores inmunohistoquímicos, junto con las pruebas de imagen, nos permiten asegurar un diagnóstico adecuado. Por lo tanto, el alto grado de sospecha junto a la colaboración del otorrinolaringólogo, radiólogo y patólogo es esencial para un adecuado diagnóstico y manejo óptimo de estos pacientes.

\section{Bibliografía}

1. Silva TD, Ferreira CB, Leite GB, de Menezes Pontes JR, Antunes HS. Oral manifestations of lymphoma: a systematic review. Ecancermedicalscience. 2016;10:665. doi:10.3332/ecancer.2016.665.

2. González-Fontal GR, Rosales JD, Jaramillo R, Henao-
Martinez AF. Primary extranodal, extralymphatic hodgkin lymphoma of the mandible. Case Rep Med. 2011;2011:387570. doi: 10.1155/2011/387570.

3. Abdelwahed Hussein MR. Non-Hodgkin's lymphoma of the oral cavity and maxillofacial region: a pathologist viewpoint. Expert Rev Hematol. 2018;11(9):737-748. doi: 10.1080/17474086.2018.1506326.

4. Imai T, Michizawa M, Degami H. NK cell lymphoblastic lymphoma in the masticator space: a case of non-Hodgkin lymphoma with challenging maxillofacial manifestation and immunophenotype. Oral Surgery, Oral Med Oral Pathol Oral Radiol Endodontology. 2009;108(6):897-903. doi: 10.1016/j. tripleo.2009.07.024.

5. Chong J, Som PM, Silvers AR, Dalton JF. Extranodal non-Hodgkin lymphoma involving the muscles of mastication. Am J Neuroradiol. 1998;19(10):18491851.

6. Guastafierro S, Falcone U, Petriccione L, et al. An unusual cause of facial swelling: Primary extranodal non-hodgkin lymphoma of the masseter muscle. Am J Med Sci. 2011;341(2):160-162. doi: 10.1097/ MAJ.0b013e3181fb7136.

7. Mahima V, Guledgud M. Presentación mandibular primaria del linfoma no Hodgkin. Sociedad

Iberoamericana de Información Científica. 2013;9:2-4.

8. Liapi A, Dhanasekar G, Hock YL, East DM. An unusual case of primary extranodal non-Hodgkin's lymphoma in the muscles of facial expression. $J$ Laryngol Otol. 2006;120(3):1978-1980. doi: 10.1017/ S0022215106009480.

9. Work WP, Penner JA, Hybels RL. Lymphomas of the Masticator Space. Arch Otolaryngol. 1976;102(9):532534. doi: 10.1001/archotol.1976.00780140064005.

10. Aspestrand F, Boysen M. CT and MR imaging of primary tumors of the masticator space. Acta radiol. 1992;33(6):518-522. doi:10.1177/028418519203300603.

11. Lee VS, Martínez S, Coleman RE. Primary muscle lymphoma: clinical and imaging findings. Radiology. 1997;203(1):237-244. doi: 10.1148/ radiology.203.1.9122401.

12. Muralee Mohan C, Thakral A, Bhat SK. Primary extranodal non-Hodgkin's lymphoma involving masseter and buccinator muscles. Int J Oral Maxillofac Surg. 2012;41(11):1393-1396. doi: 10.1016/j.ijom.2011.09.025. 